\title{
Prepubertal and postpubertal vitiligo: a multivariate comparative study in 375 patients $^{*}$
}

\author{
Huma Khurrum ${ }^{1}$
}

\author{
Khalid M AlGhamdi²
}

DOI: http:/ / dx.doi.org/10.1590/abd1806-4841.20176154

\begin{abstract}
BACKGROUND: The onset of vitiligo during childhood is common. Limited data exist that compare the clinical associations of prepubertal and postpubertal vitiligo in Arabs.

OвјестіV: To compare the clinical profile of pre and postpubertal onset vitiligo.

METHODs: A cross-sectional observational study was conducted. The Vitiligo European Task Force questionnaire was completed for each patient.

RESULTS: A total of 375 patients were included; 199 had postpubertal vitiligo (>12 years), and 176 had prepubertal onset vitiligo (<12years). There were more females in the prepubertal group $(49 \%)$ than in the postpubertal group $(29 \%)$, p-value $<0.001$. The prepubertal group has had more involvement than the postpubertal group ( $45 \%$ vs $30 \%, p=0.004)$. Only 8 cases of segmental vitiligo were observed; five were observed in the prepubertal group of patients. Female gender (OR=2.3; 95\% CI:1.5, 3.5), presence of halo nevus (OR=2.2; 95\% CI:1.1, 4.4) and face involvement (OR=1.9; 95\% CI:1.2, 2.9) were positively associated with prepubertal vitiligo. Stress, as an onset factor, was positively associated (OR=0.51; 95\% CI:0.3, 0.8) with postpubertal onset vitiligo.

STUDY LIMITATIONS: A possible selection bias toward more severe vitiligo cases can be a limitation, because the study was conducted in a clinic specialized in vitiligo. Moreover, a likelihood of false recall bias cannot be excluded.

CONCLUSIONS: Our data present clinical evidence that vitiligo behaves mostly the same way in the prepubertal group as in the postpubertal group. However, female over-representation, more face involvement and more halo nevi were observed in prepubertal vitiligo, while stress was more prevalent as an aggravating factor in postpubertal vitiligo patients.
\end{abstract}

Keywords: Nevus, halo; Stress, psychological; Vitiligo

\section{INTRODUCTION}

Vitiligo is mainly an acquired depigmentary disorder affecting around $1 \%$ of the world's population. ${ }^{1-3}$ In $25 \%$ of the cases, it begins prior to 14 years of age. ${ }^{4}$

The mean age of onset varied from 4 to 8 years among different studies. The mean age of onset in Caucasians is 24 years. ${ }^{5-7}$ Vitiligo can occur in infants as young as 3 months old. Congenital vitiligo has been described previously. ${ }^{8}$ Prepubertal vitiligo may differ from postpubertal vitiligo.

To date, few reports have addressed differences between true paediatric vitiligo/prepubertal onset vitiligo (before the age of 12) and later-onset vitiligo (after the age of 12 , postpubertal onset vitiligo). . $^{3,9,10}$

There is an inadequate number of studies comparing various associated clinical factors with both prepubertal and postpubertal vitiligo forms. The present study shows the results of a cross-sectional observational study, after having received ethical clearance from the institutional board review of King Khalid University hospital, aimed at identifying factors associated with prepubertal and postpubertal onset vitiligo using univariate and multivariate logistic regression analyses.

\section{METHODS}

This research conducted an observational cross-sectional study in the dermatology clinic at King Khalid University Hospital between January 1, 2008, and July 1, 2012. Ethics committee approval was obtained from the university's review board. All patients with vitiligo, defined as an acquired progressive depigmentation according to the Vitiligo European Task Force (VETF) definition, were enrolled in this study. ${ }^{11}$

The VETF questionnaires were completed by each vitiligo patient attending the clinic on his/her first visit. ${ }^{12}$ The VETF form provided a wide range of demographic and clinical information, in-

Received on 13.06.2016.

Approved by the Advisory Board and accepted for publication on 09.08.2016.

* Work performed at the King Khalid university hospital.

Financial support: The project was financially supported in part by King Saud University, Vice Dean of Research Chairs.

Conflict of interest: None.

Vitiligo Research Chair, College of Medicine, King Saud University, Riyadh, Saudi Arabia.

Dermatology Department, College of Medicine, King Saud University, Riyadh, Saudi Arabia.

(C2017 by Anais Brasileiros de Dermatologia 
cluding sex, age, age at onset of vitiligo, phototype, site of involvement and distribution patterns, Koebner phenomenon (KP, defined in the VETF grid as depigmentation on scars), presence of halo nevi, family history of vitiligo, personal and/or family history of chronic autoimmune/autoinflammatory diseases (thyroid disease, atopy, rheumatoid arthritis, psoriasis, type 1 diabetes mellitus, alopecia areata or inflammatory bowel disease), family history of premature greying of hair (more than $50 \%$ of white hair before the age of 40 ), emotional stress at onset and response to treatment, if any.

\section{Statistical analysis}

Patient characteristics were summarized as counts and percentages, and were compared between prepubertal onset $(\leq 12$ years) and postpubertal onset vitiligo ( $>12$ years) using a chi-squared test. Logistic regression analysis was used to study the effect of risk factors on the age at onset of prepubertal vitiligo, and odds ratios with $95 \%$ confidence intervals were computed. The analyses were performed without adjustments for each of the potential risk factors and were further adjusted by entering clinically important covariates in the adjusted multivariable model. All p-values were considered to be statistically significant at the 5\% level. Hosmer and
Lemeshow goodness-of-fit was used to test the adequacy of the logistic regression model. Statistical analyses were performed using STATA statistical software version 13 (StataCorp. 2013. Stata Statistical Software: Release 13. College Station, TX: StataCorp LP).

\section{RESULTS}

\section{Demographic and clinical characteristics of patients}

A total of 375 patients were included, of which 176 (male 90, female 86) were in the prepubertal group, and 199 (male 140, female 59) were in the postpubertal vitiligo onset group (Table 1). More males were observed in the postpubertal group $(70.4 \%$ vs. $51 \%$ ), while females were observed more in the prepubertal group than in the postpubertal group ( $49 \%$ vs. $29.6 \%$ ), p value $<0.001$. Disease durations of $\leq 3$ years were $37.2 \%$ and $39.4 \%$ in the prepubertal and postpubertal groups, respectively. Emotional stress as an aggravating factor was observed more in the postpubertal group $(24.1 \%)$ when compared to the prepubertal group $(9.7 \%)$. The presence of one or more halo nevi was observed more often $(13.1 \%)$ in the prepubertal group when compared to the postpubertal group $(6.5 \%)$. With regards to the previous episode of spontaneous regimentation, there was a significant difference of $9.7 \%$ and $19.6 \%$, respectively, in

TABLE 1: Description of patient characteristics according to the age of vitiligo onset ( $\leq 12$ years vs $>12$ years) $(n=375)$

Demographic and clinical data

Male

Female

Duration of disease $(n=370)$

$\leq 3 y$

$>3 y$

Koebner phenomenon $(\mathrm{n}=371)$

Yes

No

Emotional stress as onset factor $(n=375)$

Triggering

Aggravating factor

Has no relation

Presence of halo nevus $(n=375)$

None

One or more

Previous episode of repigmentation $(n=375)$

None

Spontaneous

Following Rx

After sun exposure

Type of vitiligo $(n=370)$

Focal

Segmental

Acrofacial

Generalised vitiligo

Mucosal

Mixed

\section{Prepubertal onset of vitiligo Postpubertal onset of vitiligo} ( $>12$ years) $n=199$

( $\leq 12$ years), $n=176$

$\mathrm{n}$

90

86

64

108

47

128

31

17

128

153

23

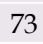

17

$\%$

51.1

48.9

37.2

62.8

26.9

73.1

17.6

9.7

72.7

86.9

13.1

41.5

9.7

47.7

1.1

20.2

2.8

21.4

42.2

0.5

12.7
140

59

78

120

58

138

\section{6}

48

115

186

13

78

39

82

0

53

3

34

77

5

25 p

$\%$

70.4

29.6

39.4

60.6

29.6

70.4

18.1

24.1

57.8

93.5

6.5

39.2

19.6

41.2

0 
the prepubertal and postpubertal groups. The prepubertal vitiligo group is associated with a family history of premature greying of hairs $(\mathrm{p}=0.05)$.

No significant difference was observed between a family history of vitiligo or autoimmune disease with respect to the vitiligo onset in either group. The personal history of autoimmune disease was similar in both groups (Table 2). Generalised vitiligo was the predominant type of disease observed during consultation for both groups. Facial involvement was identified more often in the prepubertal group (44.9\%) when compared to the postpubertal group (30.2\%).

\section{Logistic Regression Analysis}

Results of univariate logistic regression of very early onset for clinically important patient characteristics are presented in table 3. Female gender (OR=2.3; 95\% CI:1.5, 3.5), presence of halo nevus (OR=2.2; 95\% CI:1.1, 4.4) and face involvement (OR=1.9; $95 \%$ CI:1.2, 2.9) were positively associated with early-onset vitiligo. Stress as an onset factor was positively associated (OR=0.51; $95 \%$ CI:0.3, 0.8) with postpubertal onset vitiligo. No significant difference was observed in the distribution of previous episodes of spontaneous repigmentation, disease duration, Koebner's phenomenon, personal and family history of premature greying of hair, and family history of vitiligo and/or autoimmune disease.

When all of the patient characteristics were included simultaneously in the logistic regression analysis, female gender $(\mathrm{OR}=2.4$; 95\% CI:1.6, 3.9, $\mathrm{P}<0.001)$, stress as an aggravating factor $(\mathrm{OR}=0.45$; 95\% CI:0.3,0.7, $\mathrm{P}=0.001$ ), halo nevus ( $\mathrm{OR}=2.4$; 95\% CI:1.1, 5.4, $\mathrm{P}=0.03$ ) and face involvement $(\mathrm{OR}=1.8 ; 95 \% \mathrm{CI}: 1.1,2.8, \mathrm{P}=0.01)$ remained statistically significant (Table 4 ). The logistic regression model results using Hosmer-Lemeshow goodness-of fit were good $(p=0.77)$.

\section{DISCUSSION}

The purpose of this study was to compare prepubertal and postpubertal vitiligo in clinical and epidemiologic settings. In our study, a female gender preference was noted for prepubertal vitiligo. Other studies also report a female preponderance of $57 \%^{5-7}$ to $63 \%$, and in our study, this percentage was even higher $(66 \%)$. Other studies express equal numbers of patients for both genders. ${ }^{3,5}$ Whether girls are overrepresented in childhood, compared with late-onset vitiligo, is also ambiguous. We found a significant difference in the female:male ratio between prepubertal and postpubertal vitiligo.

Childhood vitiligo was reported to vary from adults by

TABLE 2: Description of other patient characteristics according to the age of vitiligo onset ( $\leq 12$ years versus $>12$ years ) ( $n=375$ )

\begin{tabular}{|c|c|c|c|c|c|}
\hline & \multicolumn{2}{|c|}{$\begin{array}{c}\text { Prepubertal onset of vitiligo } \\
\text { ( } \leq 12 \text { years) } n=176\end{array}$} & \multicolumn{2}{|c|}{$\begin{array}{c}\text { Postpubertal onset of vitiligo } \\
\text { ( } \leq 12 \text { years) } n=199\end{array}$} & \multirow[t]{2}{*}{$\mathbf{p}$} \\
\hline & $\mathrm{n}$ & $\%$ & $\mathrm{n}$ & $\%$ & \\
\hline Personal history of autoimmune disease $(n=375)$ & & & & & 0.44 \\
\hline Yes & 16 & 9.1 & 23 & 11.6 & \\
\hline No & 160 & 90.9 & 176 & 88.4 & \\
\hline Family history of premature greying of hair $(n=375)$ & & & & & 0.05 \\
\hline Yes & 28 & 15.9 & 19 & 9.6 & \\
\hline No & 148 & 84.1 & 180 & 90.4 & \\
\hline Family history of vitiligo $(n=371)$ & & & & & 0.47 \\
\hline Yes & 86 & 49.1 & 89 & 45.4 & \\
\hline No & 89 & 50.9 & 107 & 54.6 & \\
\hline Family history of autoimmune disease $(n=375)$ & & & & & 0.47 \\
\hline Yes & 51 & 28.9 & 51 & 25.6 & \\
\hline No & 125 & 71.0 & 148 & 74.4 & \\
\hline
\end{tabular}

TABLE 3: Univariate logistic regression for prepubertal $(\leq 12)$ vs postpubertal onset of vitiligo $(>12)(n=357)$

\section{Gender (female)}

Previous episode of spontaneous repigmentation (yes/no)

Presence of Koebner phenomenon (yes/ no)

Stress as an aggravating factor (yes/ no)

Disease duration ( $>3$ years $/ \leq 3$ years)

Halo nevus (yes/ no)

Family history of premature greying of hair (yes/no)

Family history of vitiligo (yes/ no)

Personal history of autoimmune disease (yes/no)

Family history of autoimmune disease (yes/no)

Face involvement (yes/no)

OR- unadjusted odds ratio; $\mathrm{CI}$ - Confidence interval

\begin{tabular}{cccc} 
OR & $\mathbf{9 5 \%} \mathbf{C I}$ & $\mathbf{N}$ & $\mathbf{P}$ \\
\hline 2.26 & $1.5-3.5$ & 375 & $<0.001$ \\
0.909 & $0.6-1.4$ & 375 & 0.65 \\
0.873 & $0.6-1.4$ & 371 & 0.56 \\
0.513 & $0.3-0.8$ & 375 & 0.003 \\
1.096 & $0.7-1.7$ & 370 & 0.67 \\
2.150 & $1.1-4.4$ & 375 & 0.035 \\
1.792 & $0.9-3.3$ & 375 & 0.06 \\
1.162 & $0.8-1.7$ & 371 & 0.47 \\
0.765 & $0.4-1.5$ & 375 & 0.44 \\
1.184 & $0.7-1.9$ & 375 & 0.47 \\
1.887 & $1.2-2.9$ & 375 & 0.003
\end{tabular}


TABLE 4: Multivariable logistic regression for prepubertal ( $\leq 12$ years) vs postpubertal onset of vitiligo $(>12$ years) $(n=357)$

\begin{tabular}{|c|c|c|c|}
\hline & AOR & $95 \% \mathrm{CI}$ & $\mathbf{P}$ \\
\hline Gender (female) & 2.47 & $1.6-3.9$ & $<0.001$ \\
\hline Previous episode of spontaneous repigmentation (yes/no) & 0.78 & $0.5-1.3$ & 0.31 \\
\hline Presence of Koebner phenomenon (yes/no) & 0.99 & $0.6-1.7$ & 0.98 \\
\hline Stress as an aggravating factor (yes/ no) & 0.45 & $0.3-0.7$ & 0.001 \\
\hline Disease duration ( $>3$ years $/ \leq 3$ years) & 1.28 & $0.8-2.1$ & 0.30 \\
\hline Halo nevus (yes/ no) & 2.41 & $1.1-5.4$ & 0.03 \\
\hline Family history of premature greying of hair (yes/no) & 1.37 & $0.7-2.6$ & 0.35 \\
\hline Family history of vitiligo (yes/ no) & 1.18 & $0.8-1.9$ & 0.46 \\
\hline Personal history of autoimmune disease (yes/ no) & 0.59 & $0.3-1.3$ & 0.18 \\
\hline Family history of autoimmune disease (yes/ no) & 1.19 & $0.7-1.9$ & 0.48 \\
\hline Face involvement (yes/no) & 1.79 & $1.1-2.8$ & 0.01 \\
\hline
\end{tabular}

AOR- Adjusted Odds ratio; $\mathrm{CI}=$ Confidence interval

showing a higher incidence in females; segmental vitiligo was more common and was less frequently linked to other systemic autoimmune and endocrine disorders. ${ }^{12}$ The present study also found more segmental cases in the prepubertal group. Regarding the preliminary presentation site of prepubertal vitiligo, our results are in agreement with previous studies that reported the head and neck area as the most common site and the upper extremities as the least common site. ${ }^{13}$ Psychological stress has been associated with vitiligo onset and progression. In one study, $44 \%$ of the patients with vitiligo referred to stress as the cause of their disease. ${ }^{14} \mathrm{~A}$ significant difference in the mean number of stressful events between patients with vitiligo and control subjects has also been reported. ${ }^{15}$ In a paediatric vitiligo population, disease onset has been connected to psychological aspects in $57 \%$ of the patients. ${ }^{16}$ Patients with postpubertal vitiligo associated stress with a statistically significant higher frequency $(p=0.003)$. Nevertheless, we cannot eliminate a recall bias in this difference, because patients with childhood vitiligo might be unable to recall stress events.

Vitiligo may be associated with other autoimmune disorders, such as alopecia areata, diabetes mellitus, pernicious anaemia, Addison's disease and thyroid disorder. One Indian study associated autoimmune disorders in $1.3 \%$ of the children with vitiligo.? Numerous authors have reported vitiligo-associated autoimmune disorders occurring solely in children suffering from NSV. ${ }^{17}$ In the study by Hann et al. ${ }^{13}$, associated autoimmune disorders were found in $3.4 \%$ of children with SV.

In our study, the prevalence of thyroid diseases was not significantly dissimilar in patients with prepubertal vitiligo when compared to patients with postpubertal disease. An interesting finding that, to the best of our knowledge, has not been reported before is that the prepubertal vitiligo group is associated with a family history of the premature greying of hair $(p=0.05)$.

Compared to the general population, patients with vitiligo have higher rates of a positive family history of the disease. ${ }^{12,18}$ In our study, $49.1 \%$ of the patients with childhood-onset vitiligo and $45.1 \%$ of the patients with later onset vitiligo had a positive family history of the disease. No discrimination was found between the two groups, as has also been reported elsewhere. ${ }^{3,9}$ This study found no disparity in the positive family history rate between patients with early versus late disease onset.

A positive family history of thyroid disease has been described in $32 \%$ to $43 \%$ of children with vitiligo, but in our study, no significant difference was observed in the family history of vitiligo in either group. ${ }^{12,19}$ A positive family history for the premature greying of hairs is more common in the prepubertal vitiligo group.

In an earlier study, a strong connection was found between halo nevi associated-non-segmental vitiligo and the premature greying of hair, suggesting that the latter may involve an autoimmune process. ${ }^{20}$ This hypothesis may be reinforced by the fact that in our population of vitiligo with prepubertal onset, halo nevus was observed more often $(p=0.03)$.

Our study found that patients with a prepubertal onset of vitiligo had a lesser chance of spontaneous repigmentation $(p=0.02)$. This may possibly support the early treatment of children with vitiligo, in which it is well-known that in vitiligo, treatment is more effective in fresh lesions as compared to older lesions. ${ }^{20}$

The limitations of this study include a possible selection bias toward more severe vitiligo cases, given that the study was conducted in a clinic specialized in vitiligo. Additionally, a possibility of false recall bias cannot be excluded, as patients with childhood vitiligo might be incapable of accurately remembering the period of disease onset. The strong points of this study consist of a large sample size, the first of its kind in the Middle East, and the advanced statistical methods used in the analysis.

\section{CONCLUSION}

Prepubertal onset vitiligo, when compared to postpubertal vitiligo, has distinct clinical features. No associations of thyroid abnormalities or atopic dermatitis were found with prepubertal or postpubertal vitiligo. Stress as an aggravating factor was observed more often in the postpubertal group, while prepubertal vitiligo patients had more face involvement. Further epidemiological studies are needed to establish the relationships of different factors; in particular, genetic studies should be taken into account for both prepubertal and postpubertal vitiligo. Future analysis of various factors are needed in order to establish the cause of vitiligo and to guide professionals toward a proper target therapy. $\square$ 


\section{REFERENCES}

1. Hann SK, Nordlund JJ, editors. Vitiligo: A Monograph on the Basic and Clinical Science. Oxford, London: Blackwell Science Ltd; 2000.

2. Howitz J, Brodthagen $\mathrm{H}$, Schwartz M, Thomsen K. Prevalence of vitiligo. Epidemiological survey on the Isle of Bornholm, Denmark. Arch Dermatol. 1977; $113: 47-52$

3. Cho S, Kang HC, Hahm JH. Characteristics of vitiligo in Korean children. Pediatr Dermatol. 2000;17:189-93.

4. Kakourou T. Vitiligo in children. World J Pediatr. 2009;5:265-8.

5. Hu Z, Liu JB, Ma SS, Yang S, Zhang XJ. Profile of childhood vitiligo in China: an analysis of 541 patients. Pediatr Dermatol. 2006;23:114-6.

6. Jaisankar TJ, Baruah MC, Garg BR. Vitiligo in children. Int $\mathrm{J}$ Dermatol. 1992:31:621-3.

7. Handa S, Dogra S. Epidemiology of childhood vitiligo: a study of 625 patients from north India. Pediatr Dermatol. 2003;20:207-10.

8. Kedward AL, Gawkrodger DJ. Congenital stable symmetrical type vitiligo in a patient whose mother developed vitiligo during pregnancy. Eur J Dermatol. 2008; $18: 353$.

9. Halder RM, Grimes PE, Cowan CA, Enterline JA, Chakrabarti SG, Kenney JA Jr. Childhood vitiligo. J Am Acad Dermatol. 1987;16:948-54.

10. Nicolaidou E, Antoniou C, Miniati A, Lagogianni E, Matekovits A, Stratigos A, et al. Childhood- and later-onset vitiligo have diverse epidemiologic and clinical characteristics. J Am Acad Dermatol. 2012;66:954-8.

11. Taïeb A, Picardo M; VETF Members. The definition and assessment of vitiligo: a consensus report of the Vitiligo European Task Force. Pigment Cell Res. 2007;20:27-35.
12. Pajvani U, Ahmad N, Wiley A, Levy RM, Kundu R, Mancini AJ, et al. The relationship between family medical history and childhood vitiligo. J Am Acad Dermatol. 2006;55:238-44.

13. Hann SK, Lee HJ. Segmental vitiligo: clinical findings in 208 patients. J Am Acad Dermatol. 1996:35:671-4.

14. Ezzedine K, Diallo A, Léauté-Labrèze C, Mossalayi D, Gauthier Y, Bouchtnei S, et al. Multivariate analysis of factors associated with early-onset segmental and nonsegmental vitiligo: a prospective observational study of 213 patients. $\mathrm{Br} \mathrm{J}$ Dermatol. 2011;165:44-9.

15. Manolache L, Benea V. Stress in patients with alopecia areata and vitiligo. J Eur Acad Dermatol Venereol. 2007;21:921-8.

16. Ezzedine K, Diallo A, Léauté-Labrèze C, Seneschal J, Boniface K, Cario-André M, et al. Pre- vs. post-pubertal onset of vitiligo: multivariate analysis indicates atopic diathesis association in pre-pubertal onset vitiligo. Br J Dermatol. 2012;167:490-5.

17. Mazereeuw-Hautier J, Bezio S, Mahe E, Bodemer C, Eschard C, Viseux V, et al. Groupe de Recherche Clinique en Dermatologie Pédiatrique (GRCDP). Segmental and nonsegmental childhood vitiligo has distinct clinical characteristics: a prospective observational study. J Am Acad Dermatol. 2010;62:945-9.

18. Barona Ml, Arrunátegui A, Falabella R, Alzate A. An epidemiologic case-control study in a population with vitiligo. J Am Acad Dermatol. 1995;33:621-5.

19. Prćić S, Duran V, Poljacki M. [Vitiligo in childhood]. Med Pregl. 2002;55:475-80.

20. Lee DY, Kim CR, Lee JH. Recent onset vitiligo on acral areas treated with phototherapy: need of early treatment. Photodermatol Photoimmunol Photomed. 2010;26:266-8.

\author{
MAILING ADDRESS: \\ Khalid M AlGhamdi \\ Vitiligo Research Chair, \\ Dermatology Department, \\ College of Medicine, King Saud University, \\ 11322 Riyadh, Saudi Arabia. \\ E-mail:kmgderm@gmail.com
}

How to cite this article: Khurrum H, AlGhamdi K. Prepubertal and postpubertal vitiligo: a multivariate comparative study in 375 patients. An Bras Dermatol. 2017;92(6): 811-5. 